LAWRENCE

LIVERMORE

NATIONAL

LABORATORY

Benchmark Imagery Project Selection of Real World Images for Creating Composite Images of Facilities

J. W. Goforth, L. R. Gaines, W. T. White, P. A. Pope

March 27, 2013 


\section{Disclaimer}

This document was prepared as an account of work sponsored by an agency of the United States government. Neither the United States government nor Lawrence Livermore National Security, LLC, nor any of their employees makes any warranty, expressed or implied, or assumes any legal liability or responsibility for the accuracy, completeness, or usefulness of any information, apparatus, product, or process disclosed, or represents that its use would not infringe privately owned rights. Reference herein to any specific commercial product, process, or service by trade name, trademark, manufacturer, or otherwise does not necessarily constitute or imply its endorsement, recommendation, or favoring by the United States government or Lawrence Livermore National Security, LLC. The views and opinions of authors expressed herein do not necessarily state or reflect those of the United States government or Lawrence Livermore National Security, LLC, and shall not be used for advertising or product endorsement purposes.

\section{Auspices Statement}

This work performed under the auspices of the U.S. Department of Energy by Lawrence Livermore National Laboratory under Contract DE-AC52-07NA27344. 


\title{
Benchmark Imagery Project Selection of Real World Images for Creating Composite Images of Facilities
}

\author{
John W. Goforth ${ }^{1}$, Lucinda R. Gaines ${ }^{2}$ \\ W. Travis White $\mathrm{III}^{1}$, Paul A. Pope ${ }^{2}$ \\ ${ }^{1}$ Lawrence Livermore National Laboratory \\ ${ }^{2}$ Los Alamos National Laboratory
}

March 27, 2013

\section{OVERVIEW}

The "Benchmark Imagery" project is a DOE NA-22-funded effort whose three-year goal is to create a suite of imagery (both real and composite) that can be used to test geospatial algorithms used for extracting semantic content from overhead images of industrial facilities. A composite image is an image that contains both real elements such as photographic imagery and synthetic elements such as 3D models. Composite images have the advantage of being customizable and distributable, and they can contain complete ground truth. It is our goal to generate composite images which appear realistic to both human and algorithm.

When generating composite images of facilities we use real overhead imagery to increase realism and decrease effort. To construct all elements with purely synthetic textures would require too much effort and still may not look as realistic as a real image. We use real time 3D rendering software "SceneWorks"[2][3] for rendering composite images. In SceneWorks, a scene is constructed by placing 3D models of facility components on top of a base terrain. A base terrain is a 3D model constructed from digital elevation mapping (DEM) data and then textured with overhead orthorectified imagery. The base terrain is constructed to be geospatially accurate using a real location's source data (DEM and Imagery.) This document outlines the selection criteria in selecting appropriate location and source data for constructing the base terrain.

Imagery should have resolution equal or better than desired composite image resolution. The imagery should have no seams. Seams can occur when two or more different imagery data sets exist in a single area with neither set covering the entire area. Elevation data (DEM) resolution generally should be 1/9 arc second or better depending on the variability of elevation. The imagery must contain a flat clearing of land large enough for multiple facility types. There should be no high-rise buildings anywhere in the base terrain. 


\section{BACKGROUND}

The "Benchmark Imagery" project is a DOE NA-22-funded collaborative effort between Los Alamos National Laboratory (LANL) and the Lawrence Livermore National Laboratory (LLNL). The threeyear goal is to create a suite of imagery that can be used to test geospatial algorithms used for extracting semantic content from overhead images of industrial facilities (e.g., auto-annotation). Although a variety of image modalities are used to capture overhead photographs of terrestrial facilities, the current focus of the effort of this project is conventional visual-band (RGB or RGB/NIR) images. Presently, the set of images consists of over 200 airborne images of various US industrial facilities. We have annotated all of the images in the imagery set, using a constrained vocabulary of about a dozen general terms such as "building," "tower," or "electrical substation." We are generating composite images to add to the benchmark imagery set. The composite images are being annotated as well.

Composite and synthetic images have numerous advantages over real satellite images. First and foremost, their content in a synthetic image is configurable. Creating facilities, objects, and environment in a synthetic image can be dictated by the end user and their needs. This is very important when testing algorithms on situations where imagery is limited. Facilities can be generated in different stages of construction. Images can be "captured" at different angles and different time of day. Effects such as fog, clouds, and lighting can be simulated. Sensor and mission characteristics such as over-exposure, distortion, blur, flight patterns can be modeled. Composite images are also more distributable than real images because they tend to be less sensitive in nature (unclassified). Since a site may be fictitious, it is easier to distribute to universities and other non-governmental entities. The base imagery used is always from a non-sensitive location. Composite imagery can contain complete ground truth. Since the scene is constructed in a computer, the world is completely known. The image can be segmented automatically. The exact locations of all objects are known. This is extremely helpful to algorithm developers for testing and development.

\section{METHODOLOGY}

Our goal is to create composite images which are similar to real images in the Benchmark Imagery suite. Composite images contain geospatial metadata in the same format as the real world images in the suite (in the GeoTIFF[1] file format.) The composite images should be generated with a variety of base terrain environments to test algorithms over a range of inputs. Different base terrains are essential to preventing algorithms from being mistakenly "tuned" to specific locations or environments. However, there is a trade-off between having a large variety of backgrounds and the effort required to find and populate those backgrounds with synthetic 3D models of industrial facilities. As a tractable compromise, we have decided to use 4 separate base terrains. Each base terrain will support multiple facility types, including nuclear power plants and coal-fired power plants, the two types of facility on which our synthetic images focus. 
The four different environments we selected to use as background images include sites in arid, coastal, forest, and urban/suburban areas. The arid environment, we previously selected from a region near Kettleman City, CA. We found our coastal background images by performing a limited manual search of the United States coast line. We selected a region near Galveston, TX. For the forest background we searched the main forested areas of the continental U.S. and chose a region near Ellington, MO. For the urban background, we searched the Environmental Protection Agency website for superfund/brownfield areas that would provide an adequate clearing for a facility in an urban setting, and we selected an area near Commerce City, CO.

When selecting a location to use as source data for building a base terrain there are several desirable and undesirable characteristics that must be considered. The location must contain a flat clearing of land large enough for multiple facility types (at least $100 \mathrm{~m} \times 100 \mathrm{~m}$.) The clearing should be flat in elevation (DEM) and have imagery clear of any discernable objects that would prevent a facility from being placed (built) there. The clearing should have no trees, buildings, or vehicles. Base imagery may contain shadows being cast from nearby trees and small buildings, but the shadows simulated in SceneWorks should match the underlying imagery. Finding the date that the imagery was taken can help in generating shadows that match, as SceneWorks generates shadows from facility 3D models using accurate ephemeris modeling. With the imagery date, real-time shadow rendering in SceneWorks is used to find the correct time of day that the generated shadows match the underlying imagery.

Google Earth[4] is very useful tool for seeking out and evaluating different locations. It displays high resolution imagery and DEM, and it is quickly navigated. Once a potential location is found in Google Earth and appears to be viable, the source data (DEM and imagery) should be acquired and evaluated. Most imagery in Google Earth is commercial and restricted in distribution. We acquire our source data using the USGS National Map Viewer and Download Platform[5]. Using the USGS website we acquire a $5 \mathrm{~km} \times 5 \mathrm{~km}$ region of imagery and DEM centered on the desire location of the facility.

\section{IMAGES CONSIDERED}

Figures 1, 2 and 3 illustrate examples of images that we have considered and rejected because they do not meet the criteria defined above in our methodology. Figure 1 has an elevation profile from the DEM source data which creates a slope that is too steep to easily place a facility. This does not mean that a facility could not be constructed at this location. It means it would require ground leveling which requires additional effort in $3 \mathrm{D}$ construction that is we do not desire.

Figure 2 contains a clearing of land which is only 90 meters wide in some areas. Clearings should be more than 100 meters. Figure 3 must be rejected because it has a seam in the upper right hand corner. When source data was acquired for this location there was no single dataset of high resolution imagery that covered the entire portion. 


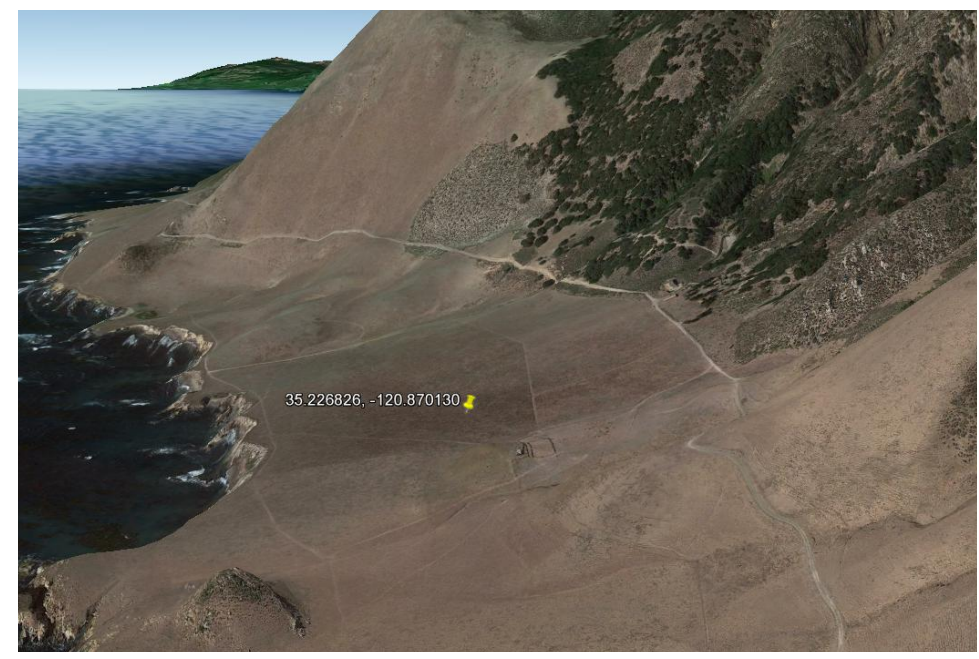

Figure 1 - REJECTION: too sloped, clearing is not flat enough.

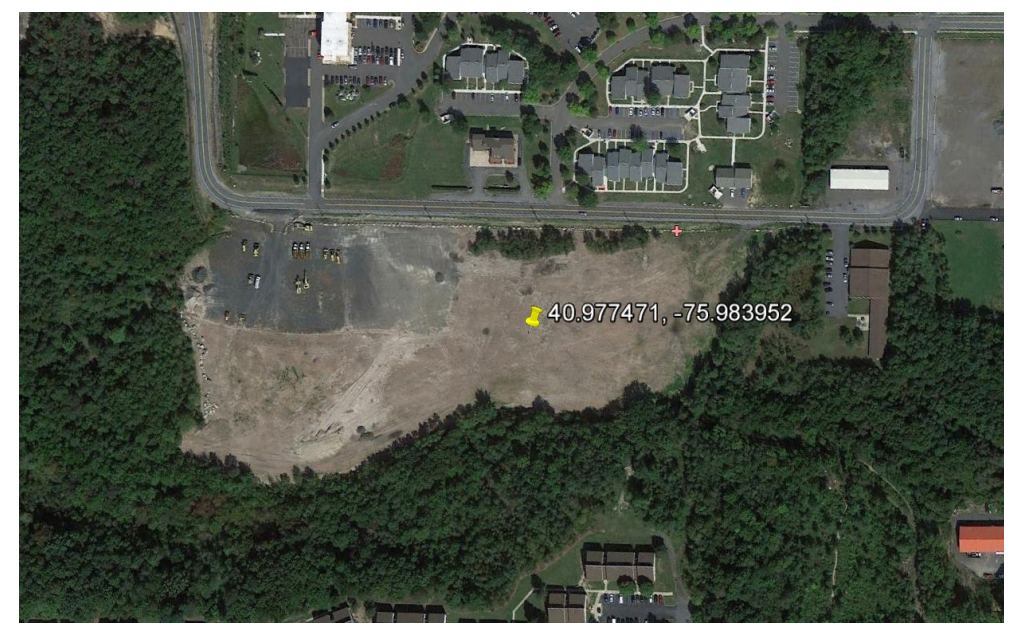

Figure 2 - REJECTION: Clearing is not large enough to support all facility types and layouts. 


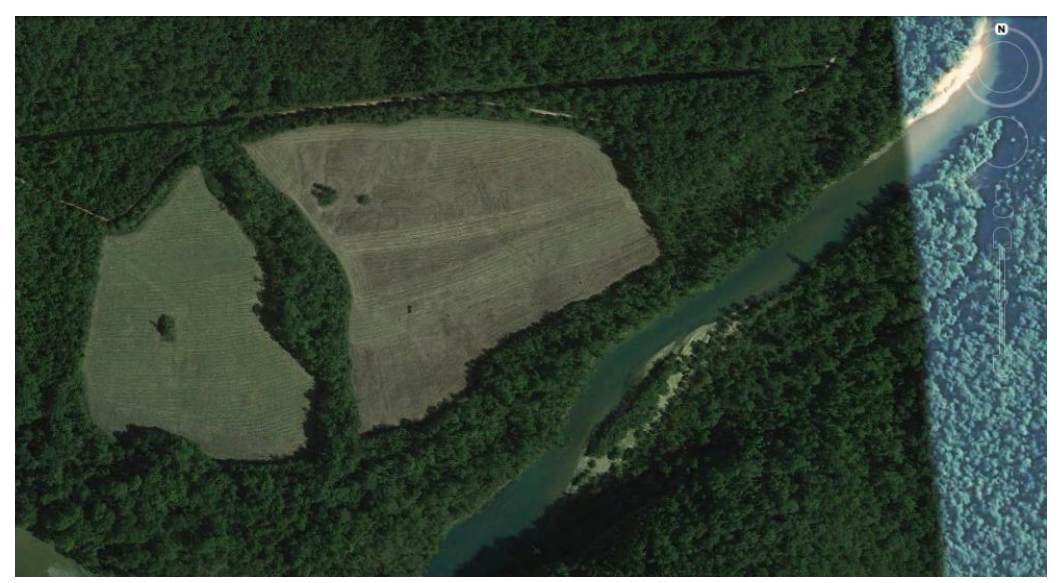

Figure 3-REJECTION: Image seam from different collections. Complete imagery from single source not available.

Figures 4, 5, 6 and 7 below are the images we have selected for generating our composite images. They comprise the 4 different environments: arid, forest, coastal, and urban/suburban. All imagery was acquired from the USGS and is freely distributable.

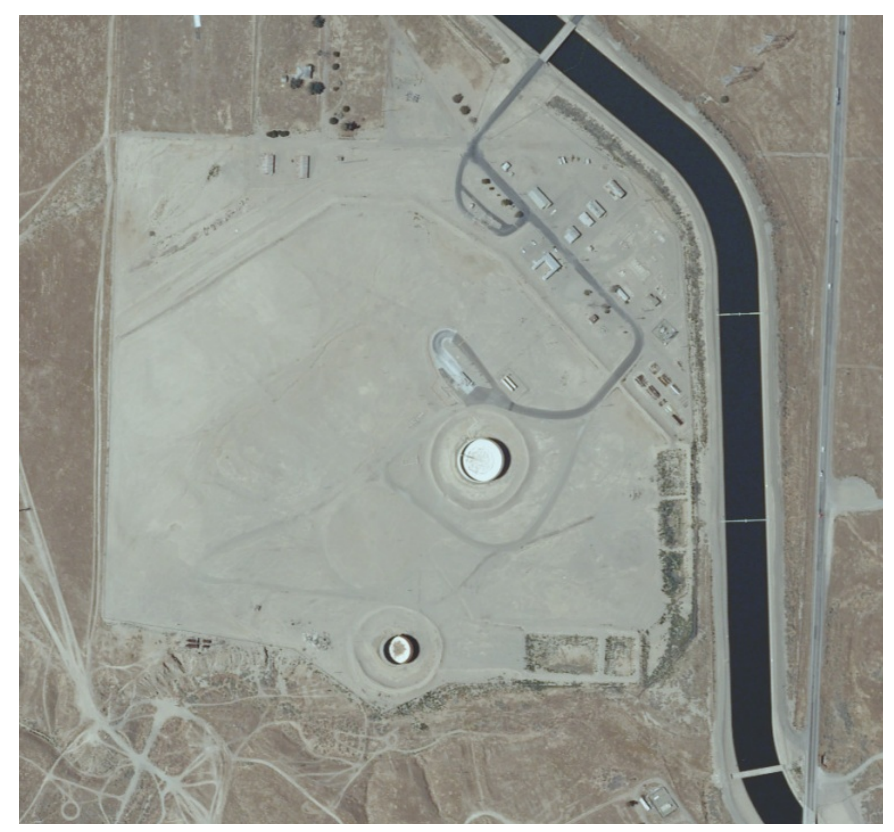

Figure 4 - Selected base image for arid environment 


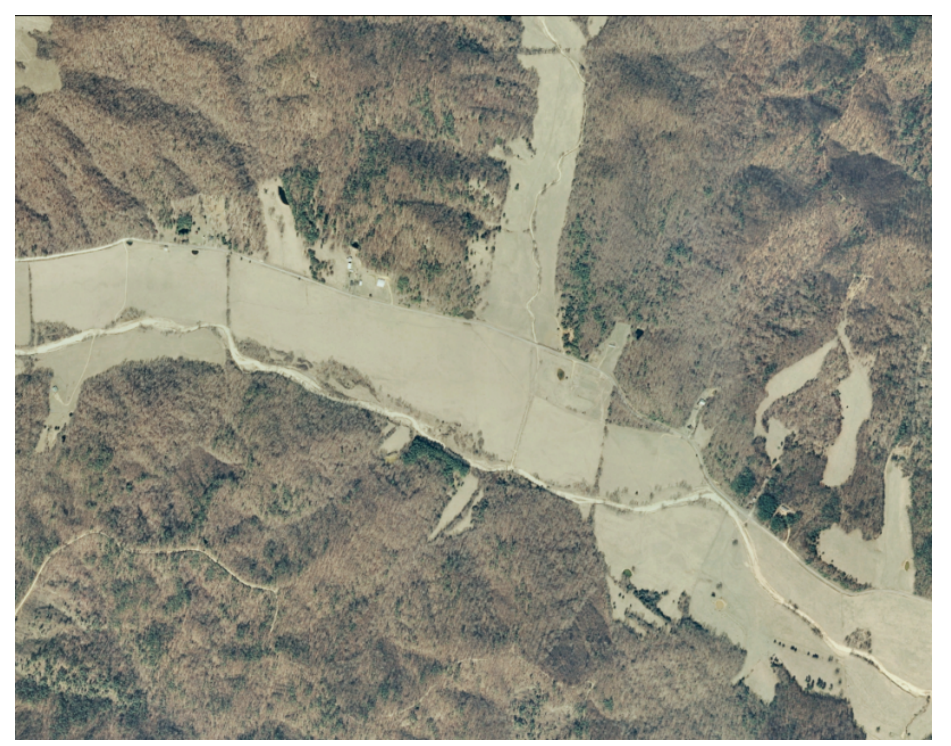

Figure 5 - Selected base image for forest environment.

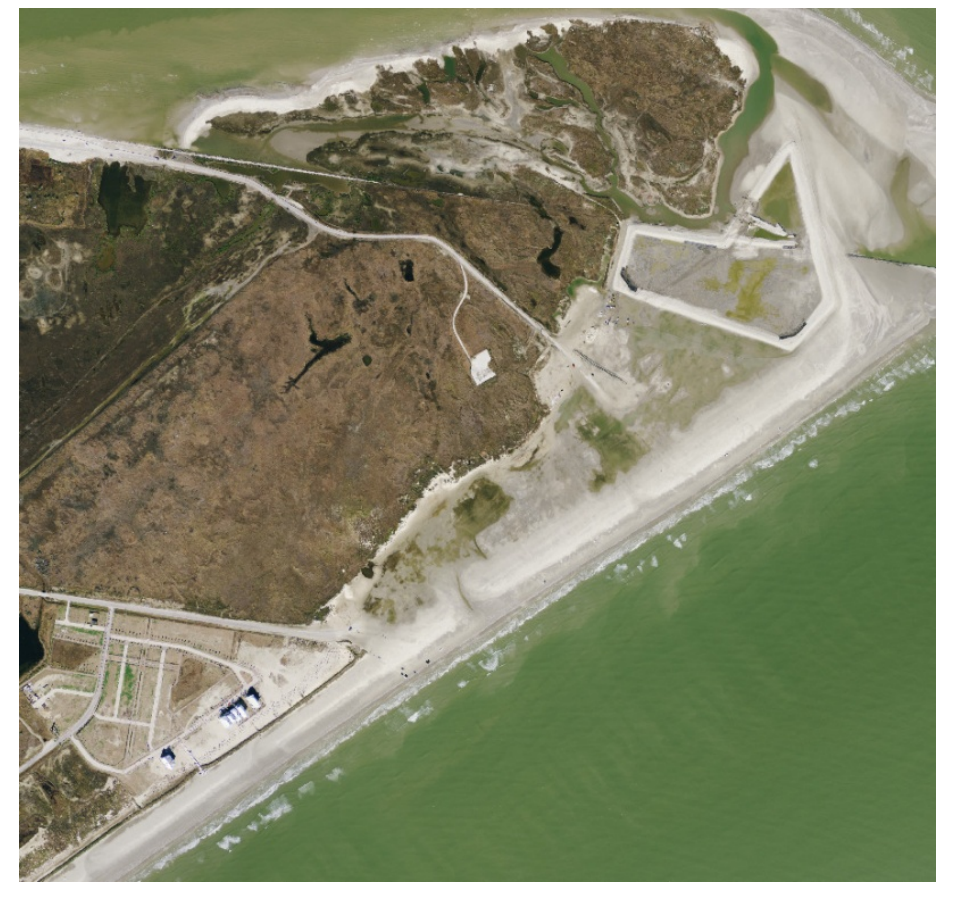

Figure 6 - Selected base image for coastal environment 


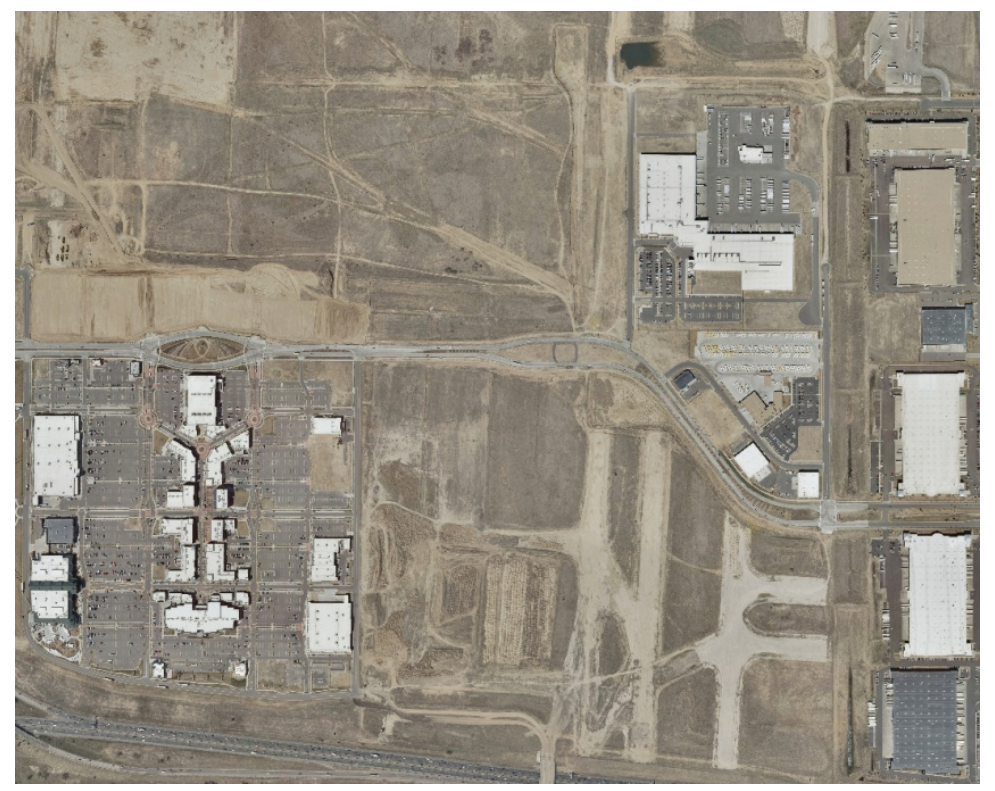

Figure 7 - Selected image for urban/suburban environment.

\section{CONCLUSION \& PATH FORWARD}

We have outlined the requirements that we use in selecting real world images for synthetically generated composite imagery. We've selected composite imagery for four different environments: arid, forest, coastal and urban/suburban. DEM has also been acquired for these four locations. The locations, imagery, and DEM meet all requirements outlined in this document. All data acquired is freely distributable.

Shown below (figure 8 and 9) is an example of how the selected image (figure 4) can be used in composite scene with a nuclear power plant. The oblique view (figure 9) highlights the detail of the lighting and model. It also shows how base imagery is draped over the DEM to provide relief in the underlying terrain (as can be seen in the hills in the background.) The nadir view (figure 8) is more typical of the composite images that we will be generating for the Benchmark Imagery Set. 


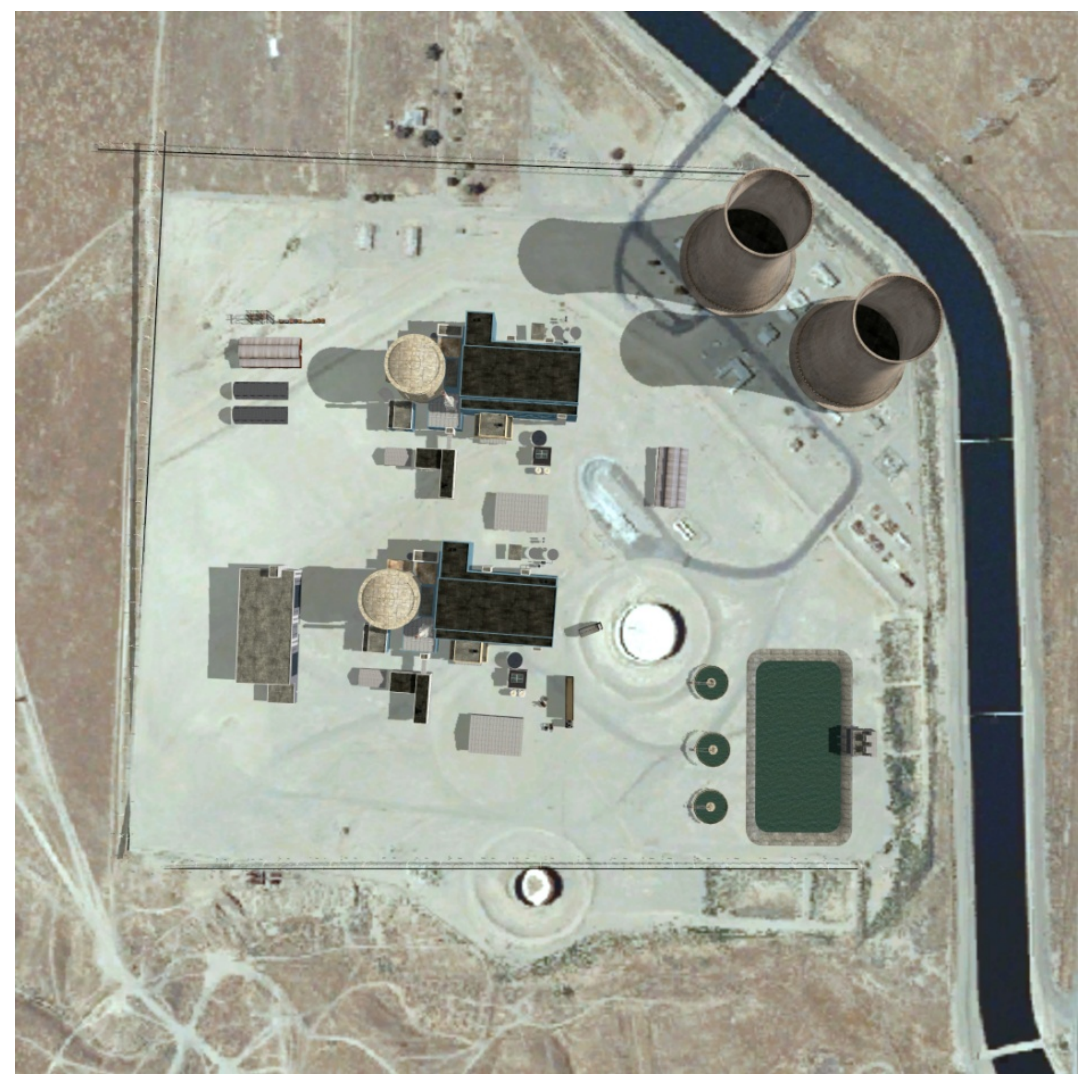

Figure 8 - Composite image (nadir view)

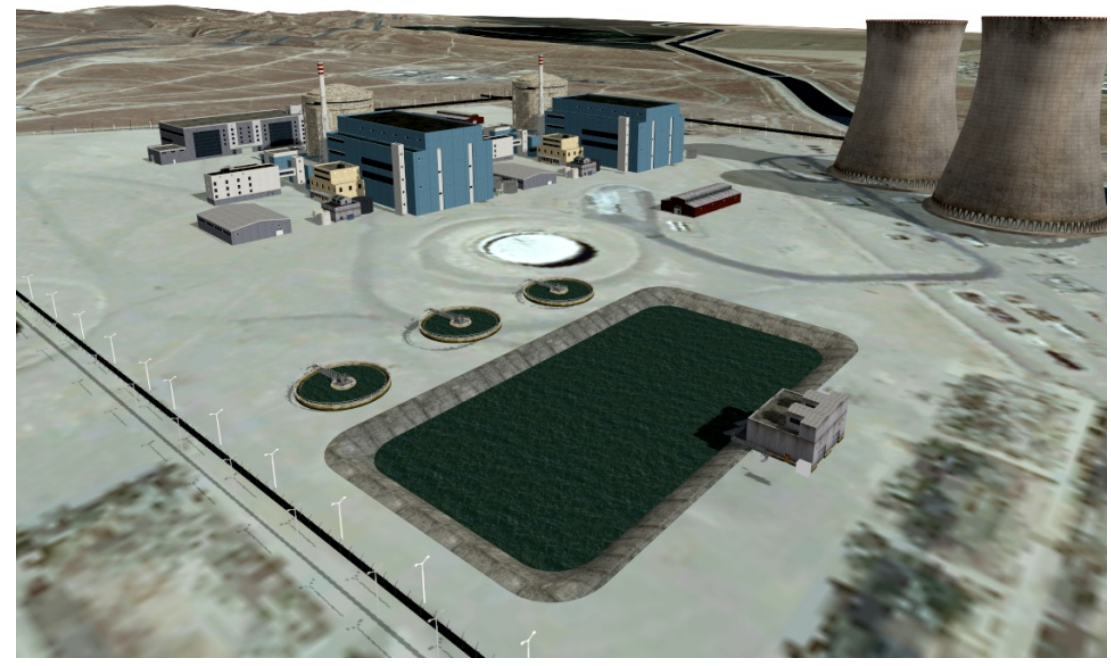

Figure 9 - Composite image (oblique view.) 


\section{References}

1. http://www.remotesensing.org/geotiff/faq.html

2. "Predicting Pollutant Dispersion and Exposure in Mixed Outdoor-Indoor-Vehicle Environments" Jeffery Peterson, US Army Dugway Proving Ground, http://cbdstconf2010. sainc.com/parallelsessionview2. aspx?conferencesessionid=22

3. "Joint Expeditionary Collective Protection System Performance Model (JECP SPM) Overview" Gaurang Dave, Naval Surface Warfare Center, Dahlgren, http://cbdstconf2010.sainc.com/parallelsessionview2.aspx?conferencesessionid=22

4. http://www.google.com/earth/index.html

5. The USGS National Map Viewer and Download Platform http://nationalmap.gov/viewer.html 